\title{
Curative effectiveness and safety of osimertinib in the treatment for non-small-cell lung cancer: a meta-analysis of the experimental evidence
}

This article was published in the following Dove Press journal: OncoTargets and Therapy

\author{
Peng Chen ${ }^{1, *}$ \\ Fuchao Chen ${ }^{2, *}$ \\ Jiexin $\mathrm{Lei}^{3}$ \\ Benhong Zhou' \\ 'Department of Pharmacy, Renmin \\ Hospital of Wuhan University, \\ Wuhan, Hubei 430060, People's \\ Republic of China; ${ }^{2}$ Department of \\ Pharmacy, Dongfeng Hospital, Hubei \\ University of Medicine, Shiyan, Hubei \\ 442008, People's Republic of China; \\ ${ }^{3}$ Department of Endocrinology, \\ Renmin Hospital of Wuhan University, \\ Wuhan, Hubei 430060, People's \\ Republic of China \\ *These authors contributed equally \\ to this work
}

Background: Osimertinib is an EGFR-TKI that is selective for both EGFR-TKI-sensitizing and T790M resistance mutations in patients with non-small-cell lung cancer (NSCLC). The purpose of this study was conducting a meta-analysis to evaluate the clinical efficacy and safety of osimertinib in the treatment for NSCLC.

Methods: Using "osimertinib" as a keyword combined with "non-small-cell lung cancer" and "randomized controlled trial" as medical subject headings, the following electronic databases were searched: PubMed, EMBASE, Cochrane Library, and China National Knowledge Infrastructure. After data extraction and quality assessment of the included randomized controlled trials, the RevMan 5.3 software and R meta package were applied for meta-analysis of objective response rate (ORR), disease control rate (DCR), progression-free survival (PFS), overall survival (OS), and safety.

Results: Ten studies met our criteria and were included in the meta-analysis, with a total of 3,260 participants. The meta-analysis showed that osimertinib therapy was superior to the control therapy alone in ORR (combined $\mathrm{RR}=1.53,95 \% \mathrm{CI}$ : $0.87-2.71, P=0.14$ ), DCR (combined RR=1.07, 95\% CI: 0.79-1.44, $P=0.66$ ), PFS (combined RR=0.32, 95\% CI: 0.24-0.44, $P<0.00001$ ), and OS (combined RR=0.57, 95\% CI: $0.47-0.70, P<0.00001$ ). In addition, osimertinib led to some toxicities, and the overall prevalence of all-grade diarrhea was $40 \%$ (95\% CI: 33-47), paronychia 26\% (95\% CI: 20-33), rash 40\% (95\% CI: 34-47), dry skin 28\% (95\% CI: 23-33), and stomatitis 15\% (95\% CI: 9-23).

Conclusion: Our study showed that osimertinib demonstrated a significant improvement in the ORR, DCR, PFS, and OS with tolerable adverse effects for NSCLC patients. However, because of some clear limitations (heterogeneity and publication bias), these results should be interpreted with caution.

Keywords: osimertinib, NSCLC, efficacy, safety, survival, meta-analysis

\section{Introduction}

Among patients with locally advanced or metastatic non-small-cell lung cancer (NSCLC) with a mutant EGFR, the EGFR-TKIs such as gefitinib, erlotinib, and afatinib are recommended as the standard first-line therapy. ${ }^{1,2}$ Despite high initial tumor response rates to first-line EGFR-TKIs, most patients ultimately develop acquired resistance. Several common mechanisms of acquired resistance have been observed in recent studies, including EGFR Thr790Met resistance mutation, MET amplification, HER2 amplification, small-cell histological transformation, and epithelial to mesenchymal transition. ${ }^{3,4}$ It has been confirmed that the EGFR Thr790Met point
Correspondence: Benhong Zhou Department of Pharmacy, Renmin Hospital of Wuhan University, Wuhan, Hubei 430060, People's Republic of China Tel +86 I53 3589843 I

Email benhongzh@whu.edu.cn 
mutation (EGFR T790M) can be detected in $\geq 50 \%$ of the patients taken after acquired resistance. ${ }^{5}$

Until recently, there were several limitations on the treatment options in post-EGFR-TKI second-line setting, with low proportions of response to platinum-based doublet chemotherapy and monochemotherapy. ${ }^{6}$ In addition, there was no global standard of care for the later-line therapy when patients experienced a failure of both EGFR-TKI therapy and platinum-based doublet chemotherapy; current treatment regimens for the same population are generally limited to monochemotherapy, rechallenge with the EGFR-TKIs, or experimental drugs in clinical trials. ${ }^{7,8}$

Osimertinib (Tagrisso, AZD9291; AstraZeneca plc) is an oral, potent, third-generation, irreversible EGFR-TKI that is selective for both EGFR-TKI-sensitizing and EGFR T790M resistance mutations, with a lower activity against wild-type EGFR. In previous studies, clinical activity and a manageable toxicity profile have been found in patients with T790Mpositive NSCLC and acquired resistance to EGFR-TKIs. ${ }^{9}$ It has been reported that osimertinib used as a second-line treatment has shown superior efficacy in NSCLC patients as compared with platinum chemotherapy in recent researches. On the basis of positive results from the clinical program, the US Food and Drug Administration (FDA) approved that osimertinib is worldwide for the treatment of patients with metastatic T790M-positive NSCLC, following progression during or after EGFR-TKI therapy. ${ }^{10,11}$

Currently, several clinical trials of osimertinib treated for NSCLC from Phase I to III have been published. However, the efficacy and safety information of these clinical studies are not identical. There has been no systematic attempt to synthesize the efficacy and safety data of this agent taking into consideration the fact that osimertinib is increasingly evaluated in NSCLC. ${ }^{12}$ Therefore, the goal of our analysis was to evaluate the clinical efficacy (overall response rate, disease control rate [DCR], progression-free survival [PFS], and overall survival [OS] rate) and safety parameters (RR and incidence of all-grade adverse events [AEs]) to provide systematical clinical evidence for use of osimertinib.

\section{Materials and methods}

\section{Search strategy}

This meta-analysis followed the Cochrane Collaboration definition and PRISMA 2009 guidelines for meta-analysis and systematic review. We searched the electronic databases of PubMed, Excerpta Medica Database (EMBASE), Cochrane Library, China National Knowledge Infrastructure (CNKI), Chinese Scientific Journals Full-Text Database (CSJFT),
Wanfang Data Knowledge Service Platform (WKSP), and the Chinese Biomedical Literature Service System (CBMdisc), and the time period for literature search was from the first available study until August 1, 2018. The keywords used in this search were as follows: "Osimertinib", "EGFR-TKIs", "non-small-cell lung cancer", "NSCLC", and "cancers" as well as "clinical trials". In addition, we also searched the abstracts that contained "osimertinib in patients with cancers" presented at the European Society for Medical Oncology (ESMO) and major meetings from the American Society of Clinical Oncology (ASCO). Finally, the references lists of original articles and review articles from the Web of Science (WOS) database were also scanned to ensure that no additional studies were missed.

\section{Study selection}

Clinical trials that met the following criteria were included: 1) prospective Phase I, II, and III clinical trials of osimertinib treatment in the patients with NSCLC; 2) reporting the data on objective response rate (ORR), DCR, PFS, and OS, as well as AEs. Exclusion criteria were the following: 1) repeat studies, abstracts, letters, reviews, editorial, or comment and 2) published against the inclusion criteria. The PICO framework guiding the development of the search strategy is shown in Table 1.

\section{Data extraction and quality assessment}

Two reviewers (Peng Chen and Fuchao Chen) independently screened the titles and abstracts of each study. The following information from each study was extracted to understand the baseline of all included studies: the first author, year of publication, number of patients enrolled in the study, therapeutic regimen, and dose of the participants. To evaluate the methodological quality of the included

Table I Eligibility criteria of the systematic review

\begin{tabular}{l|l}
\hline Category & Details \\
\hline Population & Patients with NSCLC \\
Intervention & Osimertinib \\
Comparators & Platinum+pemetrexed, standard EGFR-TKI, \\
docetaxel+bevacizumab, platinum-based \\
doublet chemotherapy, placebo \\
ORRs \\
Outcomes & PFS \\
& OS \\
& Any other efficacy outcomes \\
& Safety outcomes \\
& Prospective Phase I, II, and III trials; RCTs \\
\hline
\end{tabular}

Abbreviations: NSCLC, non-small-cell lung cancer; ORR, objective response rate; DCR, disease control rate; PFS, progression-free survival; OS, overall survival; $R C T$, randomized controlled trial. 
literature, a modified Jadad scale was used to assess the quality of the included randomized studies. The scores of high-quality studies ranged from 4 to 8 , whereas those of low-quality studies were from 0 to 3 . For non-randomized studies, the quality was assessed using Newcastle-Ottawa Quality Assessment Scale. Each study was graded as either low quality (0-5) or high quality (6-9). Any disagreements were resolved by the third author.

\section{Definition of main outcomes}

The ORR was defined as a proportion of patients having a confirmed best response of complete response or partial response as assessed by the researchers. DCR is defined as the number of patients who had a best response rating of complete response, partial response, or stable disease. The definition of PFS was that the time between the date of randomization and the date of documented progression or death, whichever occurred first. OS was calculated as the time between the first dose of study treatment and date of death. The response was evaluated by the Response Evaluation Criteria in Solid Tumors (RECIST) version 1.1 criteria, on the basis of assessment by an independent radiology review committee. For the safety analysis, we collected data about five frequent toxicity events, which included diarrhea, paronychia, rash, dry skin, and stomatitis. AEs were assessed using the National Cancer Institute Common Terminology Criteria (NCI CTC) version 4.0.

\section{Statistical analyses}

The differences between the osimertinib treatment and the control treatment were estimated by the pooled RR and HR along with 95\% CIs. The summary RR and HR estimates were conducted using a random- or fixed-effect model. Between-study heterogeneity was evaluated by $P$-value and the $I^{2}$ statistic. If $I^{2}$ was $<50 \%\left(P_{\text {heterogeneity }}>0.1\right)$, the fixedeffect model was used, if not, the random-effect model was performed. All calculations were performed by Review Manager Version 5.3 software (The Cochrane Collaboration, Oxford, UK) and R meta package (version 2.13.2) for Windows at 64 bits. $P$-values $<0.05$ or 0.01 were considered statistically significant.

\section{Results}

\section{Search results}

Overall, a total of 198 potential citations were identified according to the systematic literature searched for trials on osimertinib. Of the studies initially identified, we excluded reports that did not fulfill our inclusion criteria after first reading the titles and abstracts. Finally, our literature search yielded a total of 10 studies available for the meta-analysis, including two Phase I studies, ${ }^{16,18}$ two Phase I/II studies, ${ }^{17,22}$ one Phase II study,,$^{14}$ and five Phase III studies. ${ }^{13,15,19-21}$ The flowchart describing the trial screening and selection procedure is shown in Figure 1. Within the selected studies, there were five single-arm trails and five randomized control trials (RCTs), comprising a total of 3,260 patients. In all studies, the starting dose and schedule of osimertinib were based on US FDA guidelines ( 80 or $160 \mathrm{mg}$, orally, twice a day). The baseline characteristics of patients varied among trials, and the specific information of included trials is listed in Table 2.

\section{Statistical analysis of efficacy outcomes Comparison between osimertinib with controls alone \\ RR of ORR}

The summary RR of ORR for osimertinib vs control treatment was 1.53 (95\% CI: 0.87-2.71) using a random-effect model (heterogeneity: $\chi^{2}=48.37, \mathrm{df}=4[P=0.64], I^{2}=92 \%$; Figure 2A). As shown in Table 3, the ranking probabilities of comparison between osimertinib and control treatment from the subgroup analysis of ORR indicated that the highest RR of ORR was observed in patients associated with osimertinib vs platinum-based doublet chemotherapy (RR: 7.52 [95\% CI: 3.88-14.59], $P<0.00001)$, followed by osimertinib vs placebo (RR: 1.68 [95\% CI: $1.08-2.59$ ], $P=0.02$ ), osimertinib vs platinum combined pemetrexed (RR: 1.12
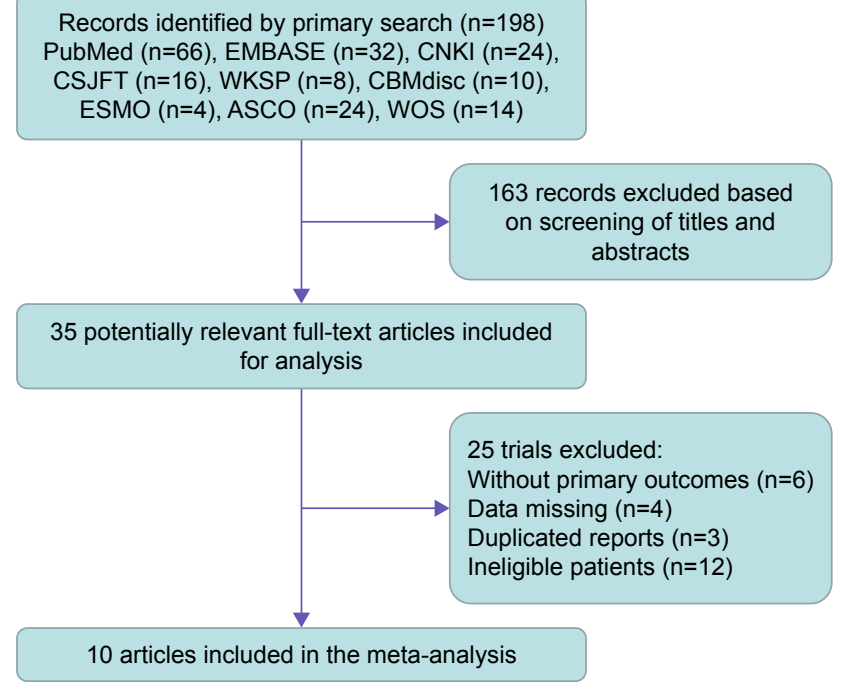

Figure I Flow diagram of the study selection process.

Abbreviations: EMBASE, Excerpta Medica Database; CNKI, China National Knowledge Infrastructure; CSJFT, Chinese Scientific Journals Full-Text; WKSP, Wanfang Data Knowledge Service Platform; CBMdisc, Chinese Biomedical Literature Service System; ESMO, European Society for Medical Oncology; ASCO, American Society of Clinical Oncology; WOS, Web of Science. 
Table 2 Baseline characteristics of trials included in the meta-analysis

\begin{tabular}{|c|c|c|c|c|c|c|c|c|c|}
\hline Study & $\begin{array}{l}\text { Study } \\
\text { type }\end{array}$ & $\begin{array}{l}\text { Tumor } \\
\text { type }\end{array}$ & $\begin{array}{l}\text { Sample } \\
\text { size, } n\end{array}$ & Treatment arms & $\begin{array}{l}\text { Primary end } \\
\text { point }\end{array}$ & $\begin{array}{l}\text { Criteria for } \\
\text { response }\end{array}$ & $\begin{array}{l}\text { Criteria } \\
\text { for AEs }\end{array}$ & Age, years & $\begin{array}{l}\text { Quality } \\
\text { score }\end{array}$ \\
\hline Mok et $\mathrm{al}^{13}$ & Phase III & NSCLC & $\begin{array}{l}279 \\
140\end{array}$ & $\begin{array}{l}\text { Osimertinib }\left(80 \mathrm{mg} \cdot \mathrm{d}^{-1}\right) \\
\text { Platinum+pemetrexed }\end{array}$ & $\begin{array}{l}\text { PFS, OR, } \\
\text { DCR, AEs }\end{array}$ & RECIST & NA & $62(25-85)$ & 5 \\
\hline Goss et $\mathrm{al}^{14}$ & Phase II & NSCLC & 210 & Osimertinib $\left(80 \mathrm{mg} \cdot \mathrm{d}^{-1}\right)$ & AEs & RECIST & NA & $64(35-88)$ & 4 \\
\hline Soria et al ${ }^{15}$ & Phase III & NSCLC & $\begin{array}{l}279 \\
277\end{array}$ & $\begin{array}{l}\text { Osimertinib }\left(80 \mathrm{mg} \cdot \mathrm{d}^{-1}\right) \\
\text { Standard EGFR-TKI }\end{array}$ & $\begin{array}{l}\text { PFS, OR, } \\
\text { DCR, AEs }\end{array}$ & RECIST & $\mathrm{NCl} \mathrm{CTC}$ & $64(26-85)$ & 6 \\
\hline $\begin{array}{l}\text { Ramalingam } \\
\text { et al }{ }^{16}\end{array}$ & Phase I & NSCLC & $\begin{array}{l}30 \\
30\end{array}$ & $\begin{array}{l}\text { Osimertinib }\left(80 \mathrm{mg} \cdot \mathrm{d}^{-1}\right) \\
\text { Osimertinib }\left(160 \mathrm{mg} \cdot \mathrm{d}^{-1}\right)\end{array}$ & OR, DCR & RECIST & $\mathrm{NCl}$ CTC & $63.5(38-91)$ & 3 \\
\hline Yang et al ${ }^{17}$ & Phase I/II & NSCLC & 201 & Osimertinib $\left(80 \mathrm{mg} \cdot \mathrm{d}^{-1}\right)$ & AEs & RECIST & $\mathrm{NCl}$ CTC & $62(37-89)$ & 3 \\
\hline Khozin et al ${ }^{18}$ & Phase I & NSCLC & 411 & Osimertinib $\left(80 \mathrm{mg} \cdot \mathrm{d}^{-1}\right)$ & AEs & RECIST & & & 3 \\
\hline Nie et $\mathrm{a}^{19}$ & Phase III & NSCLC & $\begin{array}{l}74 \\
73\end{array}$ & $\begin{array}{l}\text { Osimertinib }\left(80 \mathrm{mg} \cdot \mathrm{d}^{-1}\right) \\
\text { Docetaxel+bevacizumab }\end{array}$ & $\begin{array}{l}\text { PFS, OR, DCR } \\
\text { AEs, OS }\end{array}$ & $\begin{array}{l}\text { RECIST } \\
\text { RECIST }\end{array}$ & NA & $49.4(37-61)$ & 5 \\
\hline Mann et a $\left.\right|^{20}$ & Phase III & NSCLC & $\begin{array}{l}405 \\
61\end{array}$ & $\begin{array}{l}\text { Osimertinib }\left(80 \mathrm{mg} \cdot \mathrm{d}^{-1}\right) \\
\text { Platinum-based doublet } \\
\text { chemotherapy }\end{array}$ & $\begin{array}{l}\text { PFS, OR, } \\
\text { DCR, OS }\end{array}$ & RECIST & NA & $62(52-72)$ & 5 \\
\hline Wu et $\mathrm{a}^{21}$ & Phase III & NSCLC & $\begin{array}{l}350 \\
350\end{array}$ & $\begin{array}{l}\text { Osimertinib }\left(80 \mathrm{mg} \cdot \mathrm{d}^{-1}\right) \\
\text { Placebo }\end{array}$ & OS & NA & NA & $62.5(40-77)$ & 4 \\
\hline Jänne et $\mathrm{al}^{22}$ & Phase I/II & NSCLC & 90 & Osimertinib $\left(80 \mathrm{mg} \cdot \mathrm{d}^{-1}\right)$ & AEs & RECIST & $\mathrm{NCl}$ CTC & 60 (28-88) & 3 \\
\hline
\end{tabular}

Abbreviations: $A E$, adverse event; NSCLC, non-small-cell lung cancer; PFS, progression-free survival; DCR, disease control rate; RECIST, Response Evaluation Criteria in Solid Tumors; NA, not available; NCI CTC, National Cancer Institute Common Terminology Criteria.

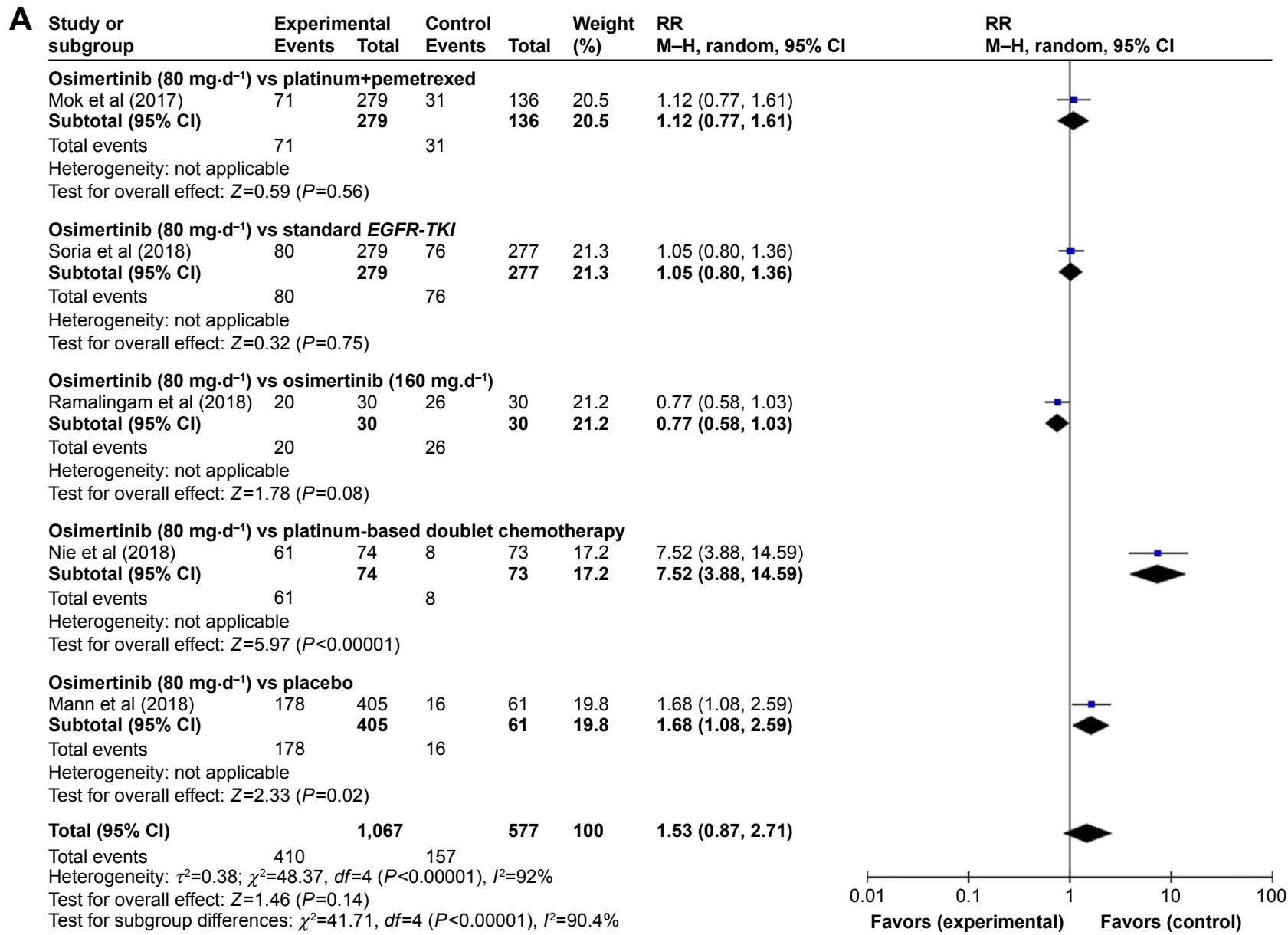

Figure 2 (Continued) 


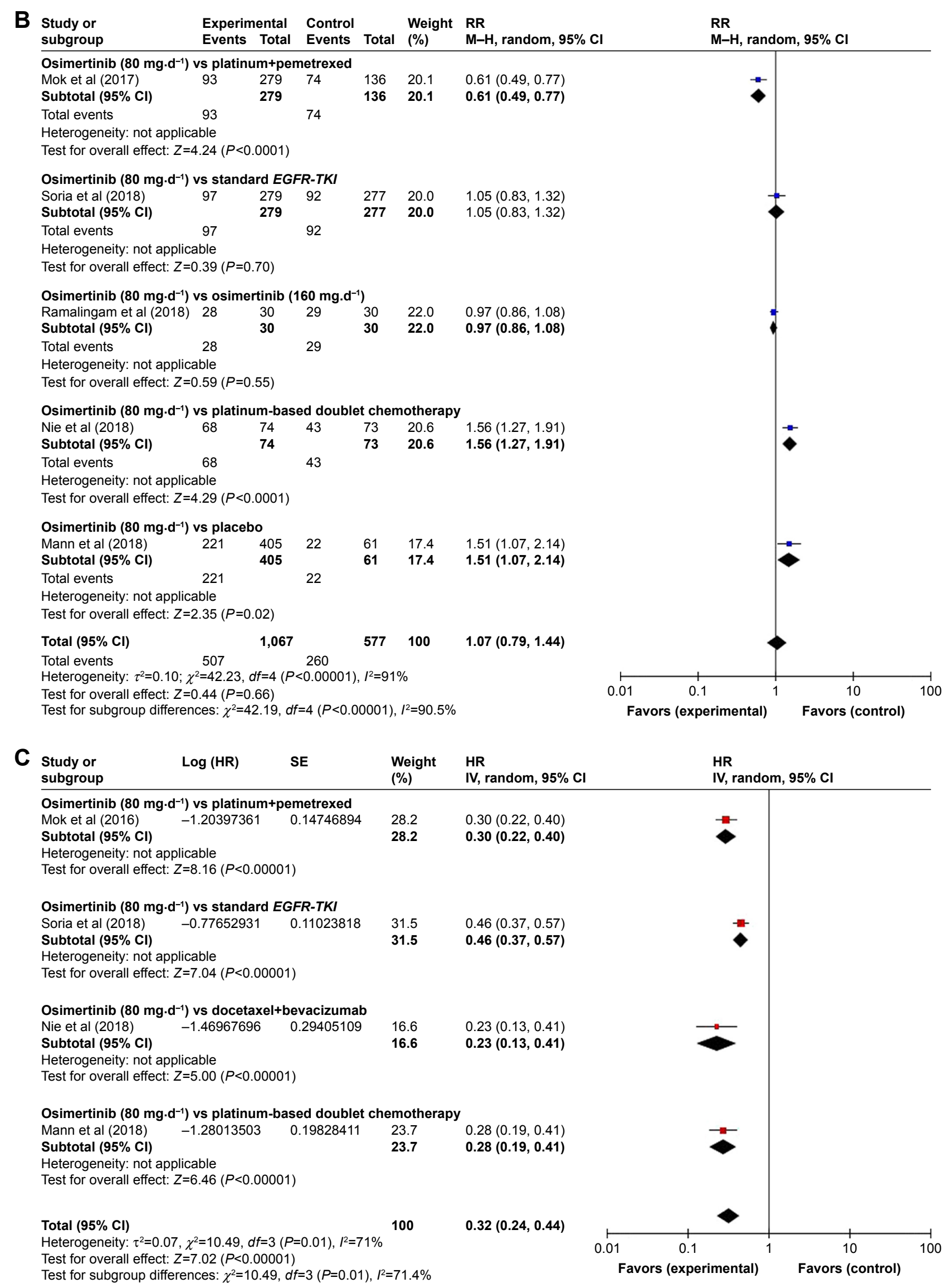

Figure 2 (Continued) 


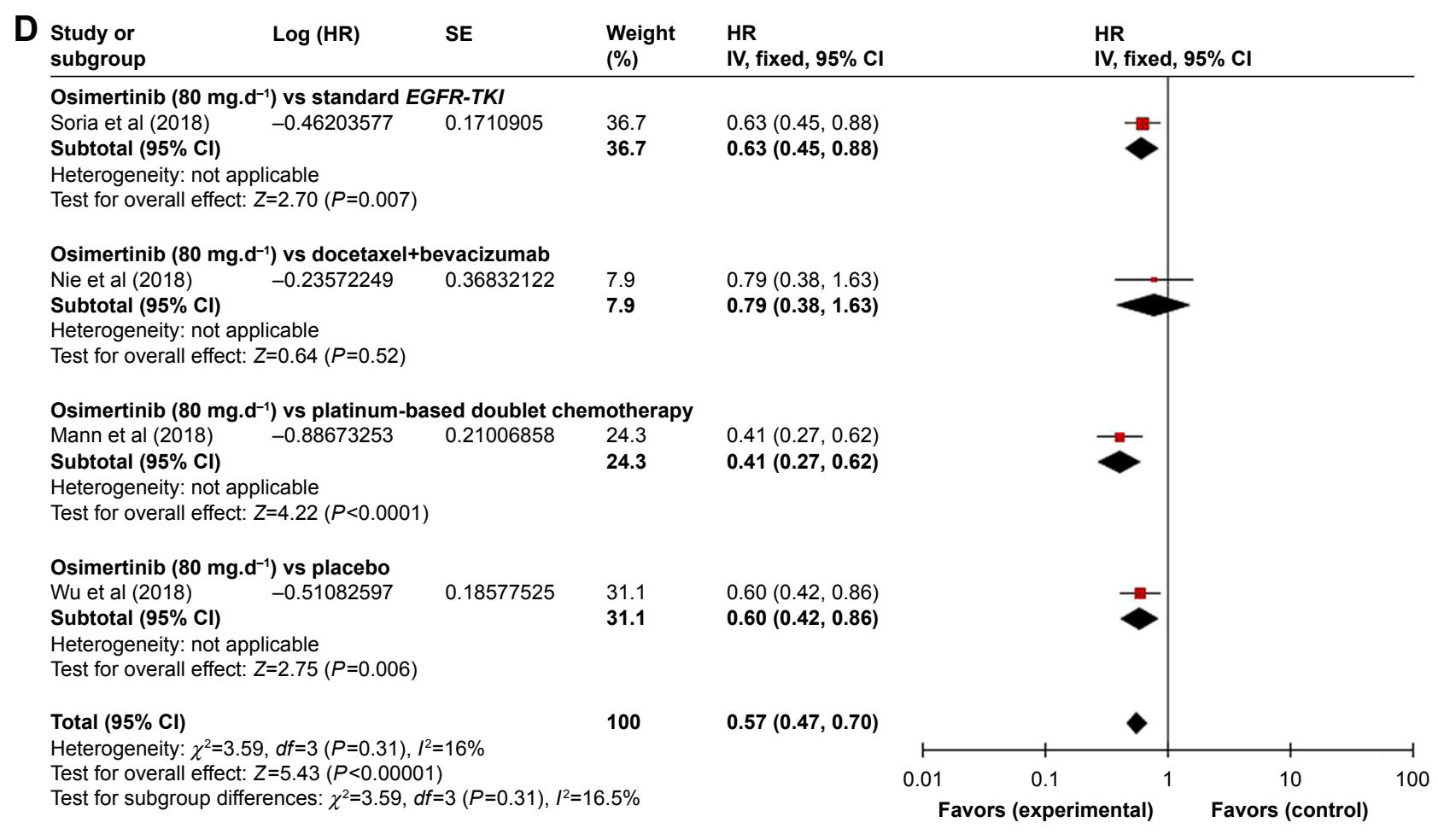

Figure 2 Forest plots analysis of the efficiency outcomes of osimertinib vs control treatment alone.

Notes: (A) ORR, (B) disease control response, (C) PFS, and (D) OS.

Abbreviations: ORR, objective response rate; PFS, progression-free survival; OS, overall survival; M-H, Mantel-Haenszel; SE, standard error; IV, intravenous.

Table 3 Outcomes of effectiveness for osimertinib in NSCLC patients

\begin{tabular}{|c|c|c|c|c|c|}
\hline \multirow[t]{2}{*}{ Efficacy outcomes } & \multirow[t]{2}{*}{ Trails } & \multirow[t]{2}{*}{ RR/HR } & \multirow[t]{2}{*}{$(95 \% \mathrm{Cl})$} & \multicolumn{2}{|c|}{ Test for overall effect } \\
\hline & & & & $Z$ value & $P$-value \\
\hline \multicolumn{6}{|l|}{ Objective response } \\
\hline Osimertinib $\left(80 \mathrm{mg} \cdot \mathrm{d}^{-1}\right)$ vs platinum+pemetrexed & 1 & 1.12 & $(0.77-1.6 I)$ & 0.59 & 0.56 \\
\hline Osimertinib $\left(80 \mathrm{mg} \cdot \mathrm{d}^{-1}\right)$ vs standard EGFR-TKI & I & 1.05 & $(0.80-1.36)$ & 0.32 & 0.75 \\
\hline Osimertinib $\left(80 \mathrm{mg} \cdot \mathrm{d}^{-1}\right)$ vs osimertinib $\left(160 \mathrm{mg} \cdot \mathrm{d}^{-1}\right)$ & 1 & 0.77 & $(0.58-1.03)$ & 1.78 & 0.08 \\
\hline Osimertinib $\left(80 \mathrm{mg} \cdot \mathrm{d}^{-1}\right)$ vs platinum-based doublet chemotherapy & I & 7.52 & $(3.88-14.59)$ & 5.97 & $<0.0000$ I \\
\hline Osimertinib $\left(80 \mathrm{mg} \cdot \mathrm{d}^{-1}\right)$ vs placebo & 1 & 1.68 & $(1.08-2.59)$ & 2.33 & 0.02 \\
\hline \multicolumn{6}{|l|}{ DCR } \\
\hline Osimertinib $\left(80 \mathrm{mg} \cdot \mathrm{d}^{-1}\right)$ vs platinum+pemetrexed & 1 & 0.61 & $(0.49-0.77)$ & 4.24 & $<0.0001$ \\
\hline Osimertinib $\left(80 \mathrm{mg} \cdot \mathrm{d}^{-1}\right)$ vs standard EGFR-TKI & I & 1.05 & $(0.83-1.32)$ & 0.39 & 0.70 \\
\hline Osimertinib $\left(80 \mathrm{mg} \cdot \mathrm{d}^{-1}\right)$ vs osimertinib $\left(160 \mathrm{mg} \cdot \mathrm{d}^{-1}\right)$ & I & 0.97 & $(0.86-1.08)$ & 0.59 & 0.55 \\
\hline Osimertinib $\left(80 \mathrm{mg} \cdot \mathrm{d}^{-1}\right)$ vs platinum-based doublet chemotherapy & I & 1.56 & $(1.27-1.91)$ & 4.29 & $<0.0001$ \\
\hline Osimertinib $\left(80 \mathrm{mg} \cdot \mathrm{d}^{-1}\right)$ vs placebo & I & 1.51 & $(1.07-2.14)$ & 2.35 & 0.02 \\
\hline \multicolumn{6}{|l|}{ PFS } \\
\hline Osimertinib $\left(80 \mathrm{mg} \cdot \mathrm{d}^{-1}\right)$ vs platinum+pemetrexed & I & 0.30 & $(0.22-0.40)$ & 8.16 & $<0.00001$ \\
\hline Osimertinib $\left(80 \mathrm{mg} \cdot \mathrm{d}^{-1}\right)$ vs standard EGFR-TKI & 1 & 0.46 & $(0.37-0.57)$ & 7.04 & $<0.00001$ \\
\hline Osimertinib $\left(80 \mathrm{mg} \cdot \mathrm{d}^{-1}\right)$ vs docetaxel+bevacizumab & I & 0.23 & $(0.13-0.4 I)$ & 5.00 & $<0.00001$ \\
\hline Osimertinib $\left(80 \mathrm{mg} \cdot \mathrm{d}^{-1}\right)$ vs platinum-based doublet chemotherapy & I & 0.28 & $(0.19-0.4 I)$ & 6.46 & $<0.0000$ I \\
\hline \multicolumn{6}{|l|}{ OS } \\
\hline Osimertinib $\left(80 \mathrm{mg} \cdot \mathrm{d}^{-1}\right)$ vs standard EGFR-TKI & I & 0.63 & $(0.45-0.88)$ & 2.70 & 0.007 \\
\hline Osimertinib $\left(80 \mathrm{mg} \cdot \mathrm{d}^{-1}\right)$ vs docetaxel+bevacizumab & I & 0.79 & $(0.38-1.63)$ & 0.64 & 0.52 \\
\hline Osimertinib $\left(80 \mathrm{mg} \cdot \mathrm{d}^{-1}\right)$ vs platinum-based doublet chemotherapy & I & 0.41 & $(0.27-0.62)$ & 4.22 & $<0.0001$ \\
\hline Osimertinib $\left(80 \mathrm{mg} \cdot \mathrm{d}^{-1}\right)$ vs placebo & 1 & 0.60 & $(0.42-0.86)$ & 2.75 & 0.006 \\
\hline
\end{tabular}

Abbreviations: NSCLC, non-small-cell lung cancer; DCR, disease control rate; PFS, progression-free survival; OS, overall survival. 
[95\% CI: 0.77-1.61], $P=0.56)$, osimertinib vs standard EGFR-TKI (RR: 1.05 [95\% CI: 0.80-1.36], $P=0.75$ ), and osimertinib ( $\left.80 \mathrm{mg} \cdot \mathrm{d}^{-1}\right)$ vs osimertinib (160 $\mathrm{mg} \cdot \mathrm{d}^{-1} ; \mathrm{RR}: 0.77$ [95\% CI: 0.58-1.03], $P=0.08$ ).

\section{$R R$ of $D C R$}

The RR of DCR was reported by five studies. ${ }^{13,15,16,20,21}$ Obvious heterogeneity $\left(\chi^{2}=42.23, \mathrm{df}=4[P<0.00001], I^{2}=91 \%\right)$ was present among the included studies. The estimated RR of osimertinib vs control treatment by the random-effects model was 1.07 (95\% CI: 0.79-1.44; Figure 2B). For the subgroup analysis of RR of DCR, osimertinib vs platinum combined pemetrexed (RR: 0.61, 95\% CI: 0.49-0.77, P<0.0001), osimertinib vs platinum-based doublet chemotherapy (RR: 1.56, 95\% CI: $1.27-0.77, P<0.0001)$, and osimertinib vs placebo (RR: $1.51,95 \% \mathrm{CI}: 1.07-2.14, P=0.02$ ) showed a significant difference, while osimertinib vs standard EGFR-TKI and osimertinib $\left(80 \mathrm{mg} \cdot \mathrm{d}^{-1}\right)$ vs osimertinib $\left(160 \mathrm{mg} \cdot \mathrm{d}^{-1}\right)$ showed no significant difference (RR: $1.05,95 \% \mathrm{CI}: 0.83-1.32$, $P=0.70$; RR: 0.97, 95\% CI: 0.86-1.08, $P=0.55$; Table 3).

\section{HR of PFS}

Four studies ${ }^{13,15,19,20}$ provided the information on PFS, and the $\mathrm{HR}$ values were explicitly reported in these studies. As shown in Figure $2 C$, the results of our random-effects $\left(\chi^{2}=10.49\right.$, $\left.\mathrm{df}=3[P=0.01], I^{2}=71 \%\right)$ meta-analysis for PFS indicated that there was a significant difference in HRs for osimertinib therapy vs control therapy (HR: 0.32 [95\% CI: 0.24-0.44], $P<0.00001$ ), which indicated a $68 \%$ reduction in the risk of disease progression in patients treated with osimertinibbased method. The results of subgroup analysis showed that osimertinib significantly prolonged PFS as compared with combination of platinum and pemetrexed (HR: 0.30, 95\% CI: 0.22-0.40, $P<0.00001$ ), standard EGFR-TKI (HR: 0.46, 95\% CI: 0.37-0.57, $P<0.00001)$, docetaxel combined bevacizumab (HR: 0.23, 95\% CI: 0.13-0.41, $P<0.00001$ ), or platinum-based doublet chemotherapy (HR: $0.28,95 \%$ CI: 0.19-0.41, $P<0.00001)$ alone.

\section{HR of OS}

Total four RCTs ${ }^{15,19-21}$ reported this outcome contributed to the analysis of OS. Heterogeneity between the four trials was $\chi^{2}=3.59, \mathrm{df}=3(P<0.00001), I^{2}=16 \%$. After an analysis with fixed-effect model, we got the result that HR: 0.57 (95\% CI: 0.47-0.70), $P<0.00001$ (Figure 2D). We also found that HR for OS in osimertinib vs standard EGFR-TKI, osimertinib vs docetaxel-bevacizumab, osimertinib vs platinum-based doublet chemotherapy, and osimertinib vs placebo were HR: 0.63 (95\% CI: 0.45-0.88), $P=0.007$; HR: $0.79(95 \%$ CI: $0.38-1.63), P=0.52$; HR: 0.41 (95\% CI: $0.27-0.62$ ),
$P<0.0001$; and HR: 0.60 (95\% CI: 0.42-0.86), $P=0.006$, respectively.

\section{Pooled ORR and DCR}

Results of the random-effect model (heterogeneity: $I^{2}=96 \%$, $P<0.0001)$ showed that the pooled ORR of the whole population of osimertinib was 0.57 (95\% CI: 0.41-0.72; Figure 3A). Six studies ${ }^{13-16,19,20}$ presented the data about DCR; the pooled rate was 0.74 (95\% CI: 0.56-0.87) with a significant heterogeneity $\left(I^{2}=97 \%, P<0.0001\right)$, which is shown in Figure 3B. A subgroup analysis has been conducted according the dosage of osimertinib treatment ( 80 and $\left.160 \mathrm{mg} \cdot \mathrm{d}^{-1}\right)$. The results of the subgroup analysis showed that the pooled rate of ORR in osimertinib $80 \mathrm{mg} \cdot \mathrm{d}^{-1}$ and osimertinib $160 \mathrm{mg} \cdot \mathrm{d}^{-1}$ was 0.87 (95\% CI: 0.69-0.95) and 0.52 (95\% CI: 0.35-0.68), respectively, and DCR was 0.97 (95\% CI: 0.80-1.00) and 0.69 (95\% CI: 0.50-0.84), respectively.

\section{Statistical analysis of safety outcomes RR of all-grade AEs}

A meta-analysis of the RR of all-grade AEs was performed on the included RCTs. Results of the random-effect or fixed-effect model showed that the pooled RR of all-grade AEs (diarrhea, paronychia, rash, dry skin, and stomatitis) with osimertinib therapy vs controls was 2.31 (95\% CI: 0.72-7.42), 3.70 (95\% CI: 0.19-72.70), 4.22 (95\% CI: 0.40-44.62), 3.98 (95\% CI: 0.67-23.47), and 1.22 (95\% CI: 0.83-1.78), respectively (Figure 4; Table 4).

The subgroup analyses for the risk of all-grade AEs have been performed according to the type of treatment (osimertinib vs platinum+pemetrexed, osimertinib vs standard EGFR-TKI, osimertinib vs docetaxel+bevacizumab, osimertinib vs platinum-based doublet chemotherapy, and osimertinib vs placebo). By the subgroup analysis of the RR of all-grade AEs for osimertinib therapy vs controls, the following were found: there was a significant difference in RRs of all-grade diarrhea associated with osimertinib vs platinum combined pemetrexed and osimertinib vs docetaxel combined bevacizumab ( $\mathrm{RR}=3.67,95 \% \mathrm{CI}: 2.23-6.04, P<0.00001$; $\mathrm{RR}=3.75,95 \% \mathrm{CI}: 2.23-6.04, P=0.005$ ), while no significant differences were observed in osimertinib vs standard EGFRTKI (RR=1.01, 95\% CI: 0.87-1.16, $P=0.94$; Figure 4A).

As for paronychia, osimertinib vs platinum combined pemetrexed showed significant results $(\mathrm{RR}=14.87,95 \% \mathrm{CI}$ : 3.69-59.90, $P=0.0001)$, whereas osimertinib vs standard EGFR-TKI showed no significant results ( $R=1.06$, 95\% CI: $0.84-1.34, P=0.63$; Figure 4B). Regarding the rash, we found that the comparison between RR of high-grade rash events was more higher in osimertinib vs docetaxel 
A Study Events Total

Proportion $95 \% \mathrm{Cl}$ W (random)

Subgroup variables=osimertinib $\left(160 \mathrm{mg} \cdot \mathrm{d}^{-1}\right)$ Ramalingam et al (2018) 26 Random-effects model $\quad 30$ Heterogeneity: $I^{2}=\mathrm{NaN} \%, \tau^{2}=0, P=1$

Subgroup variables $=0$ simertinib $\left(80 \mathrm{mg} \cdot \mathrm{d}^{-1}\right)$ Mok et al (2017)

Goss et al (2017)

Soria et al (2018)

Ramalingam et al (2018)

Nie et al (2018)

Mann et al (2018)

$71 \quad 279$

$140 \quad 210$

$80 \quad 279$

$20 \quad 30$

61

Random-effects model 178

Heterogeneity: $I^{2}=96.5 \%, \tau^{2}=0.665, P<0.0001$

Random-effects model $\quad 1,307$

Heterogeneity: $I^{2}=96.2 \%, \tau^{2}=0.7227, P<0.0001$

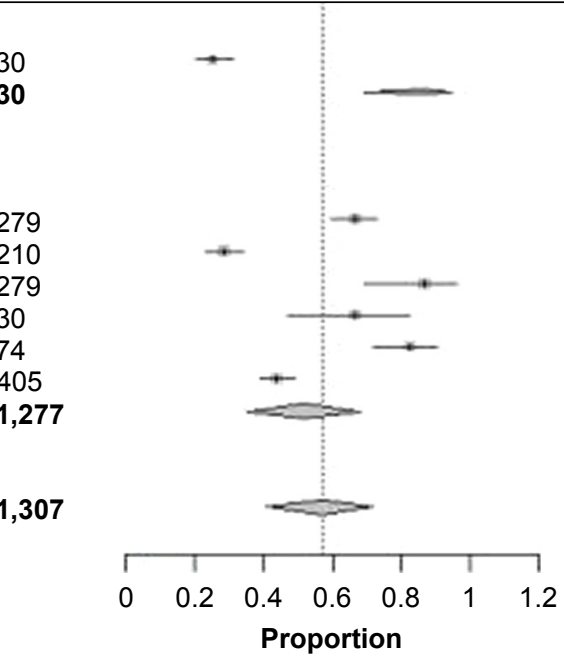

$0.25(0.20,0.31) \quad 11.3 \%$

$0.87(0.69,0.95) \quad 11.3 \%$

$0.67(0.60,0.73) \quad 15.4 \%$

$0.29(0.23,0.34) \quad 15.3 \%$

$0.87(0.69,0.96) \quad 15.4 \%$

$0.67(0.47,0.83) \quad 13.1 \%$

$0.82(0.72,0.90) \quad 14.0 \%$

$0.44(0.39,0.49) \quad 15.6 \%$

$0.52(0.35,0.68) \quad 88.7 \%$

$0.57(0.41,0.72) \quad 100 \%$

Proportion

B

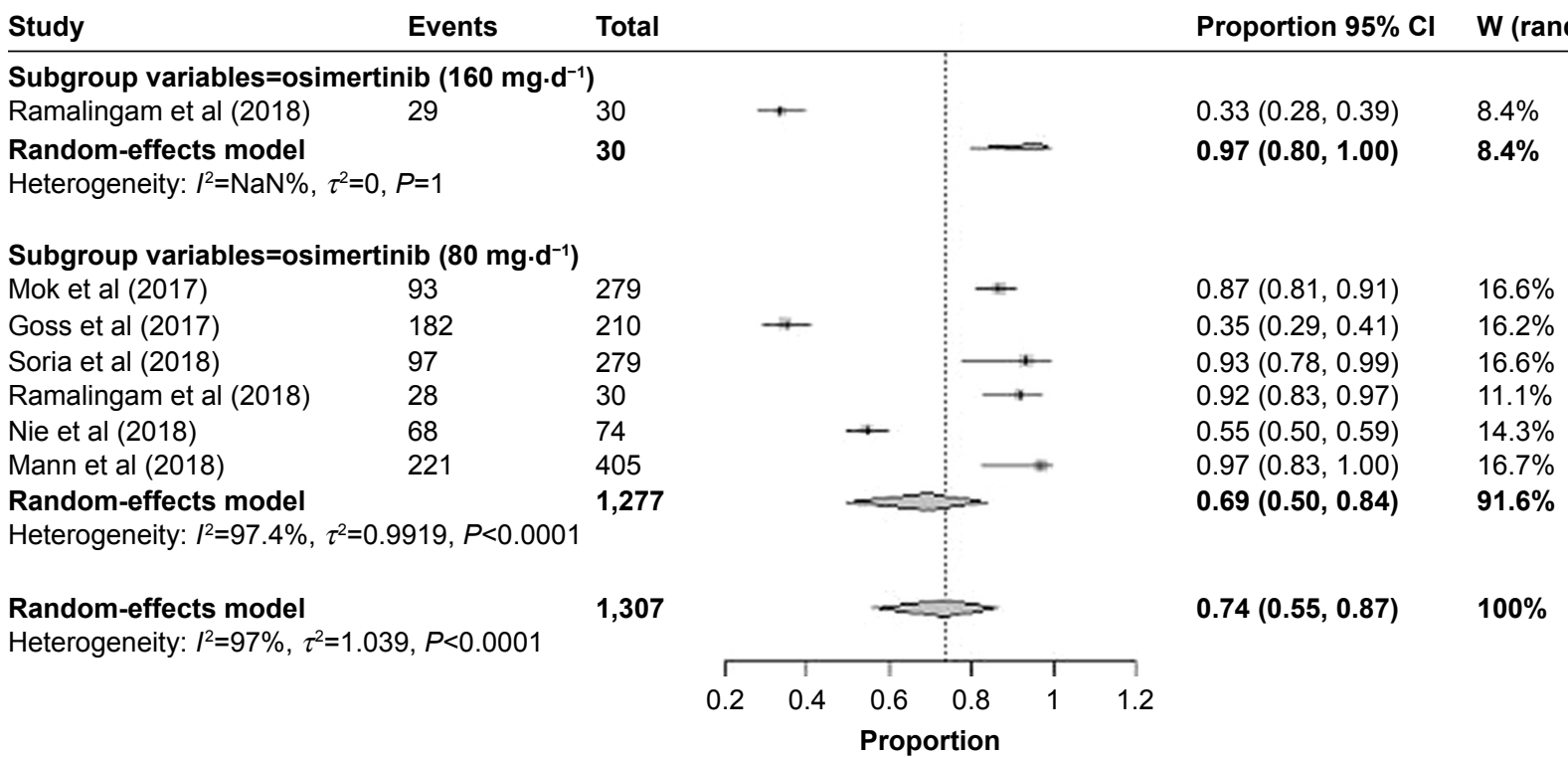

Figure 3 Forest plots analysis of pooled ORR and DCR.

Notes: (A) ORR and (B) DCR.

Abbreviations: ORR, objective response rate; $D C R$, disease control rate; $W$, weight.

combined bevacizumab ( $\mathrm{RR}=25.65,95 \%$ CI: $3.57-184.09$, $P<0.00001$ ), followed by osimertinib vs platinum combined pemetrexed and osimertinib vs standard EGFR-TKI (RR=5.73, 95\% CI: 2.87-11.44, $P<0.00001$ and $\mathrm{RR}=0.74$, 95\% CI: 0.66-0.83, $P<0.00001$, respectively; Figure 4C).

Regarding the dry skin, the RR and $95 \%$ CI for dry skin in osimertinib vs platinum combined pemetrexed and osimertinib vs docetaxel combined bevacizumab were 5.28 $(2.35-11.88)$ and $20.72(2.86-150.02)$, respectively and resulted significantly $(P<0.0001$ and $P=0.003$, respectively). However, there were no significant differences between RR of all-grade dry skin in osimertinib vs standard EGFR-TKI
(RR=0.99, 95\% CI: 0.80-1.24, $P=0.95$; Figure 4D). Finally, osimertinib vs standard EGFR-TKI showed a significant difference in all-grade stomatitis ( $R R=1.42,95 \%$ CI: $1.05-1.91$, $P=0.02)$. No significant differences were observed between osimertinib vs platinum combined pemetrexed $(\mathrm{RR}=0.95$, 95\% CI: 0.59-1.54, $P=0.84$; Figure 4E).

Incidence of all-grade AEs

In general, seven studies ${ }^{13-15,17-19,22}$ provided the data on incidence of all-grade AEs. Rates of the all-grade common AEs of osimertinib were analyzed and included diarrhea (40\%, 95\% CI: 33-47), paronychia (26\%, 95\% CI: 20-33), rash 
A study

\begin{tabular}{|c|c|c|c|c|c|c|}
\hline $\begin{array}{l}\text { Study or } \\
\text { subgroup }\end{array}$ & $\begin{array}{l}\text { Experim } \\
\text { Events }\end{array}$ & Total & $\begin{array}{l}\text { Control } \\
\text { Events }\end{array}$ & Total & $\begin{array}{l}\text { Weight } \\
(\%)\end{array}$ & $\begin{array}{l}\text { RR } \\
\text { M-H, random, } 95\end{array}$ \\
\hline \multicolumn{7}{|c|}{ Osimertinib $\left(80 \mathrm{mg}^{\cdot} \mathrm{d}^{-1}\right)$ vs platinum+pemetrexed } \\
\hline Mok et al (2017) & 113 & 279 & 15 & 136 & 34.2 & $3.67(2.23,6.04)$ \\
\hline Subtotal $(95 \% \mathrm{Cl})$ & & 279 & & 136 & 34.2 & $3.67(2.23,6.04)$ \\
\hline Total events & 113 & & 15 & & & \\
\hline \multicolumn{7}{|c|}{ Heterogeneity: not applicable } \\
\hline \multicolumn{7}{|c|}{ Test for overall effect: $Z=5.12(P<0.00001)$} \\
\hline \multicolumn{7}{|c|}{ Osimertinib $\left(80 \mathrm{mg} \cdot \mathrm{d}^{-1}\right)$ vs standard EGFR-TKI } \\
\hline Soria et al (2018) & 161 & 279 & 159 & 277 & 36.2 & $1.01(0.87,1.16)$ \\
\hline Subtotal $(95 \% \mathrm{Cl})$ & & 279 & & 277 & 36.2 & $1.01(0.87,1.16)$ \\
\hline
\end{tabular}

Total events

161

159

$277 \quad 36.2$

$1.01(0.87,1.16)$

Heterogeneity: not applicable

Test for overall effect: $Z=0.07(P=0.94)$

Osimertinib $\left(80 \mathrm{mg} \cdot \mathrm{d}^{-1}\right)$ vs docetaxel + bevacizumab

$\begin{array}{lllllll}\text { Nie et al }(\mathbf{2 0 1 8}) & 19 & 74 & 5 & 73 & 29.6 & 3.75(1.48,9.51) \\ \text { Subtotal }(\mathbf{9 5 \%} \mathbf{C l}) & & \mathbf{7 4} & & \mathbf{7 3} & \mathbf{2 9 . 6} & \mathbf{3 . 7 5}(\mathbf{1 . 4 8 , 9 . 5 1 )}\end{array}$

19

Heterogeneity: not applicable

Test for overall effect: $Z=2.78(P=0.005)$

$\begin{array}{lllll}\text { Total }(95 \% \mathrm{Cl}) & 632 & 486 & 100 & 2.31(0.72,7.42)\end{array}$

$\begin{array}{lcc}\text { Total events } & 293 & 179 \\ \text { Heterogeneity: } \tau^{2}=0.97 ; \chi^{2}=37.67, d f=2 & (P<0.00001) ; l^{2}=95 \%\end{array}$

Test for overall effect: $Z=1.41(P=0.16)$

Test for subgroup differences: $\chi^{2}=30.45, d f=2(P<0.00001) ; l^{2}=93.4 \%$

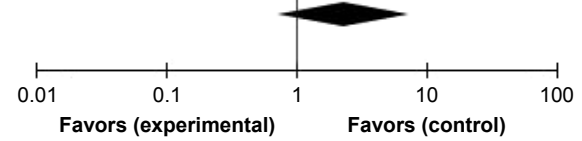

B Study or

Experimental

Osimertinib $\left(80 \mathrm{mg} \cdot \mathrm{d}^{-1}\right)$ vs platinum+pemetrexe

Events Total Events Total

Weight $\quad$ RR

(\%) M-H, random, $95 \% \mathrm{Cl}$

RR

$\begin{array}{lllllll}\text { Mok et al }(\mathbf{2 0 1 7}) & 61 & 279 & 2 & 136 & 47.3 & 14.87(3.69,59.90) \\ \text { Subtotal }(\mathbf{9 5 \%} \mathrm{Cl}) & & \mathbf{2 7 9} & & \mathbf{1 3 6} & \mathbf{4 7 . 3} & \mathbf{1 4 . 8 7}(\mathbf{3 . 6 9 , 5 9 . 9 0 )} \\ \text { Total events } & 61 & & 2 & & & \end{array}$

Total events

plicable

$14.87(3.69,59.90)$

Heterogeneity: not applicable
Test for overall effect: $Z=3.80(P=0.0001)$

Osimertinib $\left(80 \mathrm{mg} \cdot \mathrm{d}^{-1}\right)$ vs standard EGFR-TK

$\begin{array}{lllllll}\text { Soria et al }(\mathbf{2 0 1 8 )} & 97 & 279 & 91 & 277 & 52.7 & 1.06(0.84,1.34) \\ \text { Subtotal }(\mathbf{9 5 \%} \mathbf{~ C l )} & & \mathbf{2 7 9} & & \mathbf{2 7 7} & \mathbf{5 2 . 7} & \mathbf{1 . 0 6}(\mathbf{0 . 8 4}, \mathbf{1 . 3 4}) \\ \text { Total events } & 97 & & 91 & & & \end{array}$

Total events

Heterogeneity: not applicable

Test for overall effect: $Z=0.48(P=0.63)$

$\begin{array}{lllll}\text { Total }(95 \% \mathrm{Cl}) & 558 & 413 & 100 & 3.70(0.19,72.70)\end{array}$

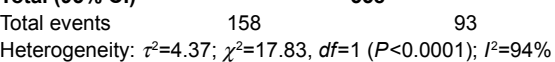

Test for overall effect: $Z=0.86(P=0.39)$

Test for subgroup differences: $\chi^{2}=13.44, d f=1(P=0.0002) ; l^{2}=92.6 \%$

C Study or

Experimental

Control

subgroup Events Total Events

Total

Weight

RR

Osimertinib $\left(80 \mathrm{mg} \cdot \mathrm{d}^{-1}\right)$ vs platinum+pemetrexed

$\begin{array}{llll}\text { Mok et al (2017) } & 94 & 279 & 8\end{array}$

Subtotal $(95 \% \mathrm{Cl})$

94

279

8

136

M-H, random, $95 \% \mathrm{CI}$

Heterogeneity: not applicable

Test for overall effect: $Z=4.94(P<0.00001)$

Osimertinib $\left(80 \mathrm{mg} \cdot \mathrm{d}^{-1}\right)$ vs standard EGFR-TKI

$\begin{array}{lllllll}\text { Soria et al }(\mathbf{2 0 1 8}) & 161 & 279 & 216 & 277 & 36.1 & 0.74(0.66,0.83) \\ \text { Subtotal }(\mathbf{9 5 \%} \mathbf{~ C l}) & & \mathbf{2 7 9} & & \mathbf{2 7 7} & \mathbf{3 6 . 1} & \mathbf{0 . 7 4}(\mathbf{0 . 6 6}, \mathbf{0 . 8 3})\end{array}$

Total events $\quad 161$

Heterogeneity: not applicable

Test for overall effect: $Z=4.99(P<0.00001)$

Osimertinib $\left(80 \mathrm{mg}^{-\mathrm{d}^{-1}}\right)$ vs docetaxel + bevacizumab

$\begin{array}{lllllll}\text { Nie et al }(2018) & 26 & 74 & 1 & 73 & 28.9 & 25.65(3.57,184.09) \\ \text { Subtotal }(\mathbf{9 5 \%} \mathbf{~ C l )} & & \mathbf{7 4} & & \mathbf{7 3} & \mathbf{2 8 . 9} & \mathbf{2 5 . 6 5}(\mathbf{3 . 5 7}, \mathbf{1 8 4 . 0 9})\end{array}$

26

74

Heterogeneity: not applicable

Test for overall effect: $Z=3.23(P=0.001)$

$\begin{array}{lllll}\text { Total }(95 \% \mathrm{Cl}) & 632 & 486 & 100 & 4.22(0.40,44.62)\end{array}$

$\begin{array}{lll}\text { Total events } & 281 & 225 \\ \text { Heterogeneity: } & \tau^{2}=4.01 ; \chi^{2}=72.16, d f=2 & (P<0.00001) ; l^{2}=97 \%\end{array}$

Test for overall effect: $Z=1.20(P=0.23)$

Test for subgroup differences: $\chi^{2}=44.61, d f=2(P<0.00001) ; l^{2}=95.5 \%$

Figure 4 (Continued) 


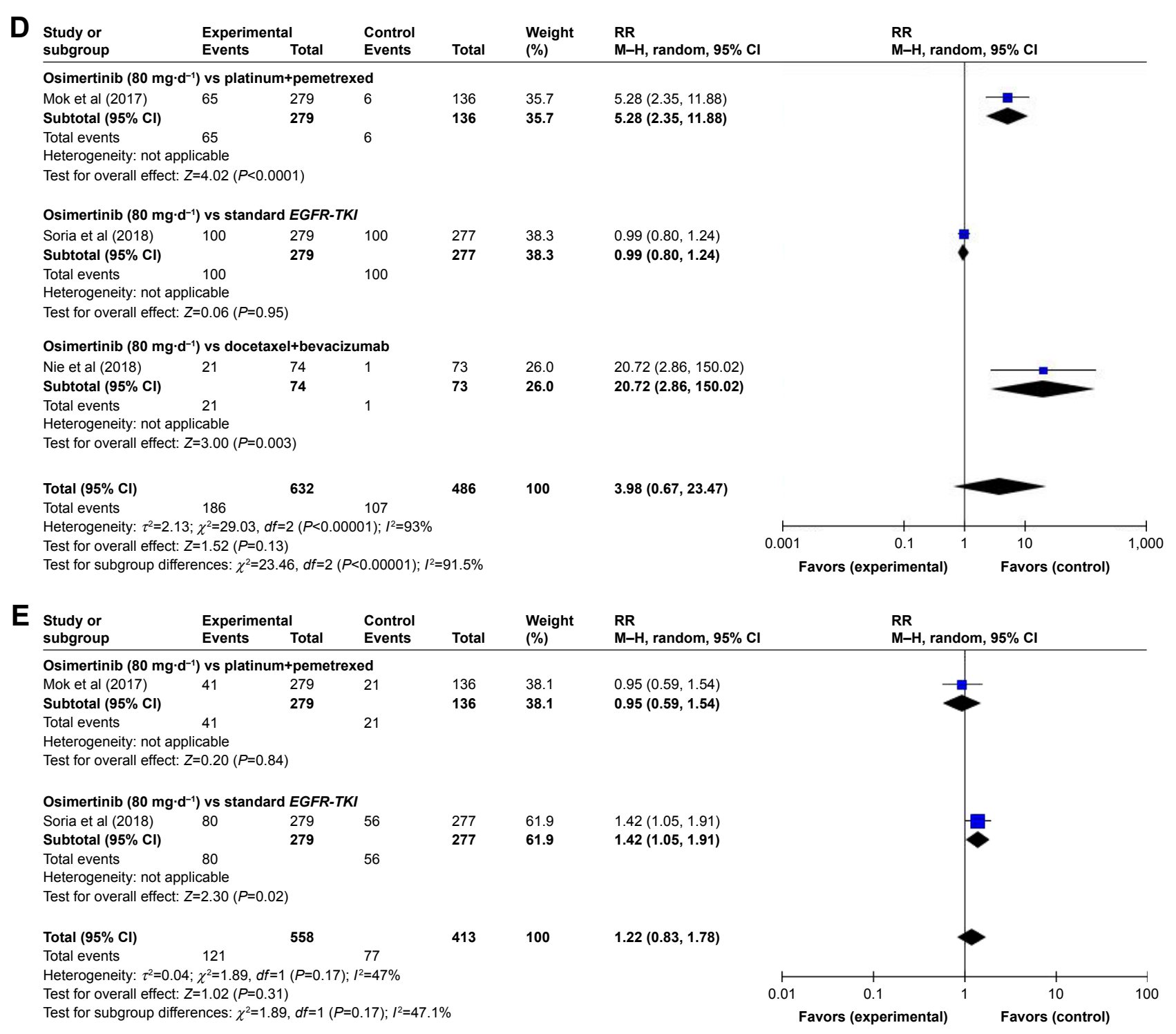

Figure 4 Subgroup analysis of the RR of all-grade AEs for osimertinib vs control treatment alone.

Notes: (A) Diarrhea, (B) paronychia, (C) rash, (D) dry skin, and (E) stomatitis.

Abbreviations: $\mathrm{AE}$, adverse event; $\mathrm{M}-\mathrm{H}$, Mantel-Haenszel.

(40\%, 95\% CI: 34-47), dry skin (28\%, 95\% CI: 23-33), and stomatitis (15\%, 95\% CI: 9-23). More details are presented in Figure 5.

\section{Publication bias}

Publication bias was assessed using Egger's funnel plot and Egger's test in this study. The funnel plot and Egger's funnel plot are displayed in Figure 6A and B. From funnel plot, it appeared a certain asymmetry, indicating that there is a certain degree of publication bias in the literature. However, the number of studies included is small, and thus, the funnel plot may not be convincing. Additionally, it was revealed that the publication bias was not significant according to the Egger's test for the incidence of all-grade AEs $(P=0.41)$.

\section{Discussion}

Prior to the approval of osimertinib, approaches to address patients with EGFR-T 790M mutation-positive NSCLC, the most common cause of acquired drug resistance in EGFRm NSCLC, have been limited by a lack of efficacy and doselimiting toxicity. Osimertinib is currently supported in North America, Europe, and Asia as a recommendable treatment for patients with metastatic NSCLC who have progressed on EGFR-targeted therapy and whose tumors harbor a T790M mutation. ${ }^{23}$ The approval was based on evidence from published randomized, open-label, international trials. ${ }^{13-22}$ Prior to summary of randomized, comparative control data for osimertinib, across different endpoints, from these studies, we performed a meta-analysis to evaluate the curative 
Table 4 Subgroup analysis of all-grade AEs for osimertinib in NSCLC patients

\begin{tabular}{|c|c|c|c|c|c|c|}
\hline \multirow[t]{2}{*}{ Subgroup } & \multicolumn{2}{|l|}{ Group } & \multirow{2}{*}{$\begin{array}{l}\text { Analysis } \\
\text { number }\end{array}$} & \multirow[t]{2}{*}{ All-grade RR } & \multirow[t]{2}{*}{$95 \% \mathrm{Cl}$} & \multirow[t]{2}{*}{$P$-value } \\
\hline & $\begin{array}{l}\text { Osimertinib } \\
\text { treatment }\end{array}$ & $\begin{array}{l}\text { Control } \\
\text { treatment }\end{array}$ & & & & \\
\hline \multicolumn{7}{|l|}{ Diarrhea } \\
\hline Osimertinib $\left(80 \mathrm{mg} \cdot \mathrm{d}^{-1}\right)$ vs platinum+pemetrexed & 279 & 136 & I & 3.67 & $2.23-6.94$ & $<0.00001$ \\
\hline Osimertinib $\left(80 \mathrm{mg} \cdot \mathrm{d}^{-1}\right)$ vs standard EGFR-TKI & 279 & 277 & I & 1.01 & $0.87-1.16$ & 0.94 \\
\hline Osimertinib $\left(80 \mathrm{mg} \cdot \mathrm{d}^{-1}\right)$ vs docetaxel+bevacizumab & 74 & 73 & I & 3.75 & $|.48-9.5|$ & 0.005 \\
\hline \multicolumn{7}{|l|}{ Paronychia } \\
\hline Osimertinib $\left(80 \mathrm{mg} \cdot \mathrm{d}^{-1}\right)$ vs platinum+pemetrexed & 279 & 136 & I & 14.87 & $3.69-59.90$ & 0.0001 \\
\hline Osimertinib $\left(80 \mathrm{mg} \cdot \mathrm{d}^{-1}\right)$ vs standard EGFR-TKI & 279 & 277 & I & 1.06 & $0.84-1.34$ & 0.63 \\
\hline \multicolumn{7}{|l|}{ Rash } \\
\hline Osimertinib $\left(80 \mathrm{mg} \cdot \mathrm{d}^{-1}\right)$ vs platinum+pemetrexed & 279 & 136 & I & 5.73 & $2.87-I I .44$ & $<0.00001$ \\
\hline Osimertinib $\left(80 \mathrm{mg} \cdot \mathrm{d}^{-1}\right)$ vs standard EGFR-TKI & 279 & 277 & I & 0.74 & $0.66-0.83$ & $<0.00001$ \\
\hline Osimertinib $\left(80 \mathrm{mg} \cdot \mathrm{d}^{-1}\right)$ vs docetaxel+bevacizumab & 74 & 73 & I & 25.65 & $3.57-184.09$ & 0.001 \\
\hline \multicolumn{7}{|l|}{ Dry skin } \\
\hline Osimertinib $\left(80 \mathrm{mg} \cdot \mathrm{d}^{-1}\right)$ vs platinum+pemetrexed & 279 & 136 & I & 5.28 & $2.35-11.88$ & $<0.0001$ \\
\hline Osimertinib $\left(80 \mathrm{mg} \cdot \mathrm{d}^{-1}\right)$ vs standard EGFR-TKI & 279 & 277 & I & 0.99 & $0.80-1.24$ & 0.95 \\
\hline Osimertinib $\left(80 \mathrm{mg} \cdot \mathrm{d}^{-1}\right)$ vs docetaxel+bevacizumab & 74 & 73 & I & 20.72 & $2.86-150.02$ & 0.003 \\
\hline \multicolumn{7}{|l|}{ Stomatitis } \\
\hline Osimertinib $\left(80 \mathrm{mg} \cdot \mathrm{d}^{-1}\right)$ vs platinum+pemetrexed & 279 & 136 & I & 0.95 & $0.59-1.54$ & 0.84 \\
\hline Osimertinib $\left(80 \mathrm{mg} \cdot \mathrm{d}^{-1}\right)$ vs standard EGFR-TKI & 279 & 277 & I & 1.42 & $1.05-1.91$ & 0.02 \\
\hline
\end{tabular}

Abbreviations: AE, adverse event; NSCLC, non-small-cell lung cancer.

effectiveness and safety of osimertinib in the treatment to provide systematical clinical evidence for targeted therapy. ${ }^{24,25}$

Osimertinib is a recommended first-line treatment for patients with metastatic EGFR Thr790Met-positive NSCLC. ${ }^{26}$ To our knowledge, this study is the first metaanalysis to report the data with a EGFR Thr790Met-directed EGFR-TKI. ${ }^{27,28}$ From our results, we found that patients with T790M-positive advanced NSCLC who received osimertinib had better ORR, DCR, PFS, and OS than did those receiving platinum therapy plus pemetrexed, standard EGFR-TKI, combination therapy of docetaxel with bevacizumab, and platinum-based doublet chemotherapy. ${ }^{29}$ Interestingly, a subgroup analysis of pooled rate of objective response and disease control showed that superior effect was found in $160 \mathrm{mg}$ osimertinib first-line treatment group than that of $80 \mathrm{mg}$ group. Although the fact was observed, there are many studies that supported approved $80 \mathrm{mg}$ once-daily dosage as the first-line therapy based on a comprehensive review of available safety, tolerability, efficacy, and pharmacokinetic data from first-and later-line patients treated with osimertinib. ${ }^{30,31}$ It has already been reported that a higher number of dose reductions as a result of AEs was observed in the $160 \mathrm{mg}$ treatment group, which is consistent with available data from later-line patients treated with osimertinib. ${ }^{32}$ Despite these advantages, osimertinib revealed some additional toxicities. Our safety results in this study were consistent with expectations from extensive previous reports. ${ }^{33}$ The most common AEs possibly treatment related to osimertinib were rash (40\%), diarrhea (40\%), dry skin (28\%), paronychia (26\%), and stomatitis (15\%). Based on our further subgroup analysis of risk of AEs, it is not difficult to find that the regimen of osimertinib $\left(80 \mathrm{mg} \cdot \mathrm{d}^{-1}\right)$ carries a lower risk in paronychia and rash compared to the standard EGFR-TKI therapy. Moreover, the subgroup analysis also showed that a more higher risk was in osimertinib vs docetaxel combined bevacizumab for diarrhea, rash, and stomatitis when compared with osimertinib vs platinum combined pemetrexed.

Mechanisms of resistance to treatment with earlygeneration EGFR-TKIs when they are used as the first-line therapy have been described previously, with EGFR T790M being the most common resistance mutation; other resistance mechanisms that have been reported include amplification of HER2, MET, and MAPK1; mutation of PIK3CA and BRAF; and small-cell transformation. ${ }^{34}$ Mechanisms of resistance to osimertinib that have also been identified in patients include KRAS amplification and acquired EGFR C797S mutation. ${ }^{35}$ Consistent with preclinical data and its mechanism of action, mechanisms of resistance to osimertinib when used as the first-line therapy remain to be fully characterized, although the result from a Phase I study showed that initial treatment with osimertinib did not result in emergence of T790M as the mechanism of drug resistance, as assessed using ctDNA from plasma samples at or after clinical progression. ${ }^{36}$ Nine patients 


\begin{tabular}{|c|c|c|c|c|c|c|}
\hline Study & Events & Total & & & Proportion $95 \% \mathrm{Cl}$ & W (random) \\
\hline \multicolumn{7}{|c|}{ Subgroup variables=diarrhea } \\
\hline Mok et al (2017) & 113 & 279 & & $\longrightarrow$ & $0.41(0.35,0.47)$ & $3.4 \%$ \\
\hline Goss et al (2016) & 70 & 210 & & + & $0.33(0.27,0.40)$ & $3.4 \%$ \\
\hline Soria et al (2018) & 161 & 279 & & $\rightarrow$ & $0.58(0.52,0.64)$ & $3.4 \%$ \\
\hline Yang et al (2017) & 86 & 201 & & $\longrightarrow$ & $0.43(0.36,0.50)$ & $3.4 \%$ \\
\hline Khozin et al (2016) & 173 & 411 & & $\rightarrow$ & $0.42(0.37,0.47)$ & $3.5 \%$ \\
\hline Nie et al $(2018)^{19}$ & 19 & 74 & & & $0.26(0.16,0.37)$ & $2.9 \%$ \\
\hline Jänne et al (2015) & 30 & 90 & & + & $0.33(0.24,0.44)$ & $3.1 \%$ \\
\hline Random-effects model & & 1,544 & & $\Rightarrow$ & $0.40(0.33,0.47)$ & $23.0 \%$ \\
\hline \multicolumn{7}{|c|}{ Heterogeneity: $I^{2}=86.5 \%, \tau^{2}=0.1286, P<0.0001$} \\
\hline \multicolumn{7}{|c|}{ Subgroup variables=dry skin } \\
\hline Mok et al (2017) & 65 & 279 & $\rightarrow$ & & $0.22(0.17,0.27)$ & $3.4 \%$ \\
\hline Goss et al (2016) & 63 & 210 & & & $0.26(0.20,0.33)$ & $3.3 \%$ \\
\hline Soria et al (2018) & 100 & 279 & & $\leftarrow$ & $0.35(0.29,0.41)$ & $3.4 \%$ \\
\hline Yang et al (2017) & 62 & 201 & & & $0.31(0.25,0.38)$ & $3.3 \%$ \\
\hline Khozin et al (2016) & 127 & 411 & 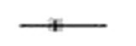 & & $0.12(0.06,0.21)$ & $3.5 \%$ \\
\hline Nie et al $(2018)^{19}$ & 21 & 74 & & — & $0.34(0.28,0.40)$ & $2.9 \%$ \\
\hline Jänne et al (2015) & 10 & 90 & & $\longrightarrow$ & $0.41(0.35,0.48)$ & $2.6 \%$ \\
\hline Random-effects model & & 1,544 & & & $0.28(0.23,0.33)$ & $22.4 \%$ \\
\hline \multicolumn{7}{|c|}{ Heterogeneity: $I^{2}=75.1 \%, \tau^{2}=0.0712, P=0.0005$} \\
\hline \multicolumn{7}{|c|}{ Subgroup variables=paronychia } \\
\hline Mok et al (2017) & 61 & 279 & & $\longrightarrow$ & $0.58(0.52,0.64)$ & $3.4 \%$ \\
\hline Goss et al (2016) & 55 & 210 & & $\longrightarrow$ & $0.40(0.33,0.47)$ & $3.3 \%$ \\
\hline Soria et al (2018) & 97 & 279 & & $\mp$ & $0.41(0.36,0.46)$ & $3.4 \%$ \\
\hline Yang et al (2017) & 63 & 201 & & & $0.35(0.24,0.47)$ & $3.3 \%$ \\
\hline Jänne et al (2015) & 11 & 90 & & - & $0.32(0.23,0.43)$ & $2.6 \%$ \\
\hline Random-effects model & & 1,059 & $\infty$ & & $0.26(0.20,0.33)$ & $16.1 \%$ \\
\hline \multicolumn{7}{|c|}{ Heterogeneity: $I^{2}=82.7 \%, \tau^{2}=0.1221, P=0.0001$} \\
\hline \multicolumn{7}{|c|}{ Subgroup variables=rash } \\
\hline Mok et al (2017) & 94 & 279 & & & $0.23(0.18,0.29)$ & $3.4 \%$ \\
\hline Goss et al (2016) & 87 & 210 & & & $0.30(0.24,0.37)$ & $3.4 \%$ \\
\hline Soria et al (2018) & 161 & 279 & & $\mp$ & $0.36(0.30,0.42)$ & $3.4 \%$ \\
\hline Yang et al (2017) & 80 & 201 & & - & $0.31(0.25,0.38)$ & $3.4 \%$ \\
\hline Khozin et al (2016) & 169 & 411 & & - & $0.31(0.26,0.36)$ & $3.5 \%$ \\
\hline Nie et al $(2018)^{19}$ & 26 & 74 & & & $0.28(0.19,0.40)$ & $3.0 \%$ \\
\hline Jänne et al (2015) & 29 & 90 & $\rightarrow$ & & $0.11(0.05,0.19)$ & $3.1 \%$ \\
\hline Random-effects model & & 1,544 & & $x$ & $0.40(0.34,0.47)$ & $23.1 \%$ \\
\hline \multicolumn{7}{|c|}{ Heterogeneity: $I^{2}=85.2 \%, \tau^{2}=0.1154, P<0.0001$} \\
\hline \multicolumn{7}{|c|}{ Subgroup variables=stomatitis } \\
\hline Mok et al (2017) & 41 & 279 & $\rightarrow$ & & $0.15(0.11,0.19)$ & $3.3 \%$ \\
\hline Goss et al (2016) & 23 & 210 & $\rightarrow$ & & $0.11(0.07,0.16)$ & $3.1 \%$ \\
\hline Soria et al (2018) & 80 & 279 & & & $0.29(0.23,0.34)$ & $3.4 \%$ \\
\hline Yang et al (2017) & 27 & 201 & $\mp$ & & $0.13(0.09,0.19)$ & $3.1 \%$ \\
\hline Jänne et al (2015) & 9 & 90 & $\longrightarrow$ & & $0.10(0.05,0.18)$ & $2.5 \%$ \\
\hline Random-effects model & & 1,059 & $\infty$ & & $0.15(0.09,0.23)$ & $15.4 \%$ \\
\hline \multicolumn{7}{|c|}{ Heterogeneity: $I^{2}=89 \%, \tau^{2}=0.3063, P<0.0001$} \\
\hline Random-effects model & & 6,750 & & & $0.30(0.26,0.34)$ & $100 \%$ \\
\hline \multicolumn{7}{|c|}{ Heterogeneity: $I^{2}=92.3 \%, \tau^{2}=0.2709, P<0.0001$} \\
\hline & & $\begin{array}{l}\Gamma \\
0\end{array}$ & 0.2 & 0.6 & 0.8 & \\
\hline
\end{tabular}

Figure 5 Forest plot analysis of incidence of all-grade AEs. Abbreviations: $A E$, adverse event; $W$, weight. 
A

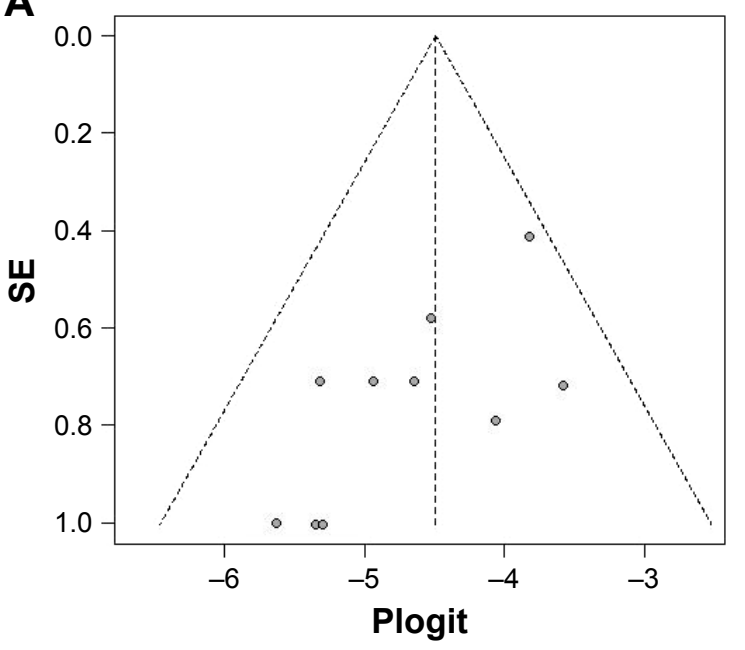

B

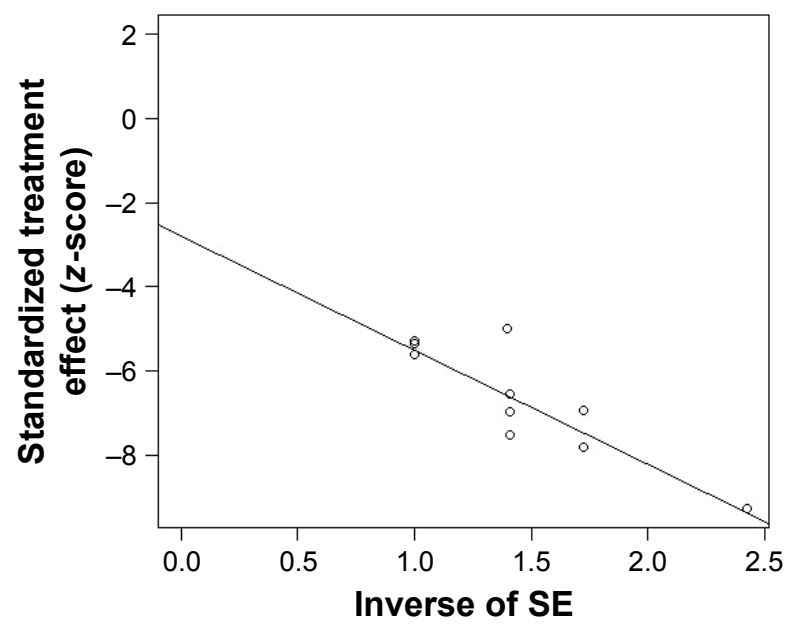

Figure 6 The funnel plot of publication bias (A) and the Egger's funnel plot of publication bias (B). Abbreviation: SE, standard error.

had putative genomic resistance mechanisms identified. Two instances of acquired C797S were identified, one in absence of a T790M mutation. This finding has potentially important clinical implications, because quinazoline-based EGFR inhibitors, including gefitinib, have been shown to effectively inhibit C797S when T790M is absent. ${ }^{37,38}$ In the report by Ramalingam et al, they identified EGFRm in 10 patients but no putative resistance mechanism at the time of progression. It is possible that molecular changes only detectable at the tissue level (eg, NSCLC transformation) and any nongenomic mechanisms of resistance were not identified in this analysis. ${ }^{16}$ It has been suggested that tissue-based analyses of resistance mechanisms will be considered as a approach to fully characterize resistance to osimertinib, and the analysis of ctDNA from identified post-progression plasma samples had involved either activation of pathways downstream of EGFR (MAPK pathway signaling) or activation of parallel signaling pathways (MET and HER2), providing the possibility of combination approaches after progression on the first-line osimertinib therapy. ${ }^{39,40}$

For the AEs associated with osimertinib, it was generally manageable with established treatment guidelines. ${ }^{41}$ Generally speaking, first, patients should be advised about the importance of managing AEs at an early stage, and the health care team of NSCLC patients should be informed to be ready for nutrition to avoid hyponatremia or hypokalemia when the gastrointestinal events, which is the most frequent AEs associated with osimertinib, occur. ${ }^{42}$ Second, it is suggested to strictly take action of dosage reduction $\left(40 \mathrm{mg} \cdot \mathrm{d}^{-1}\right)$ when grade 2 events occurred, and there may be a need to permanently discontinue medication at the onset of grade 3 or higher events. ${ }^{43}$ Third, the duration and dosage of ipilimumab or nivolumab should be based on the severity of the patient's underlying disease, recovery from immunosuppression, and clinical response. ${ }^{44}$ Moreover, in view of pharmacoeconomics, osimertinib is not covered by health insurance, and patients have to pay for the expenses of taking them all on their own. ${ }^{45}$ The average daily cost of osimertinib for adult patients is approximately $\$ 249.6438$ (at a dose of $80 \mathrm{mg}$ once daily, $80 \mathrm{mg} \times 30$ pills/box [TAGRISSO] for a month). However, further studies would be required to confirm these derived conclusions. ${ }^{46}$

\section{Limitations}

The current research also had some limitations, which need to be addressed. First and most important, the number of studies and patients included in this study is small and there was a lack of sufficient data and sample size to be reliable. Second, we did not perform subgroup analysis of high-grade events because of lack of enough information. Third, the heterogeneity among the results of the studies was evident, which significantly decreased the statistical power of the analysis. Furthermore, on the basis of our study, the efficacy and safety of osimertinib combined with other therapy were unknown. Finally, the publication bias might have been occurred, and it could not be completely reflected by funnel plot. Therefore, future additional high-quality, large-sample, multicenter, randomized controlled clinical trials are needed to resolve these limitations.

\section{Conclusion}

Based on the results of current meta-analysis, osimertinib, a molecularly targeted single agent, is favorable to improve 
the survival outcomes, including the objective response, DCRs, PFS, and OS, although it may increase the incidence of some AEs. In addition, correct estimates of treatmentrelated toxicities and the efficacy of osimertinib could be fundamental to provide appropriate guidance and conduct ongoing trials. Further RCTs were warranted to update our meta-analysis and investigate the role of osimertinib in first line for NSCLC patients.

\section{Disclosure}

The authors report no conflicts of interest in this work.

\section{References}

1. Sun C, Li S, Yang C, et al. MicroRNA-187-3p mitigates non-small cell lung cancer (NSCLC) development through down-regulation of BCL6. Biochem Biophys Res Commun. 2016;471(1):82-88.

2. Reckamp KL, Melnikova VO, Karlovich C, et al. A highly sensitive and quantitative test platform for detection of NSCLC EGFR mutations in urine and plasma. $J$ Thorac Oncol. 2016;11(10):1690-1700.

3. Dong JK, Lei HM, Liang Q, et al. Overcoming erlotinib resistance in EGFR mutation-positive lung adenocarcinomas through repression of phosphoglycerate dehydrogenase. Theranostics. 2018;8(7): $1808-1823$.

4. Lin CC, Shih JY, Yu CJ, et al. Outcomes in patients with non-small-cell lung cancer and acquired Thr790Met mutation treated with osimertinib: a genomic study. Lancet Respir Med. 2018;6(2):107-116.

5. Jakobsen JN, Santoni-Rugiu E, Grauslund M, Melchior L, Sørensen JB. Concomitant driver mutations in advanced EGFR-mutated non-smallcell lung cancer and their impact on erlotinib treatment. Oncotarget. 2018;9(40):26195-26208.

6. Xu J, Zhang Y, Jin B, et al. Efficacy of EGFR tyrosine kinase inhibitors for non-adenocarcinoma lung cancer patients harboring EGFRsensitizing mutations in China. J Cancer Res Clin Oncol. 2016;142(6): 1325-1330.

7. Dai D, Li XF, Wang J, et al. Predictive efficacy of (11)C-PD153035 PET imaging for EGFR-tyrosine kinase inhibitor sensitivity in non-small cell lung cancer patients. Int J Cancer. 2016;138(4):1003-1012.

8. Inoue A, Yoshida K, Morita S, et al. Characteristics and overall survival of EGFR mutation-positive non-small cell lung cancer treated with EGFR tyrosine kinase inhibitors: a retrospective analysis for 1660 Japanese patients. Jpn J Clin Oncol. 2016;46(5):462-467.

9. Zhang CG, Yin DD, Sun SY, Han L. The use of IncRNA analysis for stratification management of prognostic risk in patients with NSCLC. Eur Rev Med Pharmacol Sci. 2017;21(1):115-119.

10. Schoenfeld JD, Sibenaller ZA, Mapuskar KA, et al. $\mathrm{O}_{2}{ }^{-}$and $\mathrm{H}_{2} \mathrm{O}_{2}{ }^{-}$ mediated disruption of Fe metabolism causes the differential susceptibility of NSCLC and GBM cancer cells to pharmacological ascorbate. Cancer Cell. 2017;31(4):487-500.

11. Ballard P, Yates JW, Yang Z, et al. Preclinical comparison of osimertinib with other EGFR-TKIs in EGFR-mutant NSCLC brain metastases models, and early evidence of clinical brain metastases activity. Clin Cancer Res. 2016;22(20):5130-5140.

12. Zhao J, Qiao CR, Ding Z, et al. A novel pathway in NSCLC cells: miR-191, targeting NFIA, is induced by chronic hypoxia, and promotes cell proliferation and migration. Mol Med Rep. 2017;15(3): 1319-1325.

13. Mok TS, Wu Y-L, Ahn M-J, et al. Osimertinib or platinum-pemetrexed in EGFR T790M-positive lung cancer. $N$ Engl J Med. 2017;376(7): 629-640.

14. Goss G, Tsai CM, Shepherd FA, et al. Osimertinib for pretreated EGFR Thr790Met-positive advanced non-small-cell lung cancer (AURA2): a multicentre, open-label, single-arm, phase 2 study. Lancet Oncol. 2016; 17(12):1643-1652.
15. Soria JC, Ohe Y, Vansteenkiste J, et al. Osimertinib in untreated EGFRmutated advanced non-small-cell lung cancer. N Engl J Med. 2018; 378(2):113-125.

16. Ramalingam SS, Yang JC, Lee CK, et al. Osimertinib as first-line treatment of EGFR mutation-positive advanced non-small-cell lung cancer. J Clin Oncol. 2018;36(9):841-849.

17. Yang JC, Ahn MJ, Kim DW, et al. Osimertinib in pretreated T790Mpositive advanced non-small-cell lung cancer: AURA study Phase II extension component. J Clin Oncol. 2017;35(12):1288-1296.

18. Khozin S, Weinstock C, Blumenthal GM. Osimertinib for the treatment of metastatic epidermal growth factor T970M positive non-small cell lung cancer. J Cancer Res Clin. 2016;23(9):2131-2135.

19. Nie K, Zhang Z, Zhang C, et al. Osimertinib compared docetaxelbevacizumab as third-line treatment in EGFR T790M mutated nonsmall-cell lung cancer. Lung Cancer. 2018;121:5-11.

20. Mann H, Andersohn F, Bodnar C, et al. Adjusted indirect comparison using propensity score matching of osimertinib to platinum-based doublet chemotherapy in patients with EGFRm T790M NSCLC who have progressed after EGFR-TKI. Clin Drug Investig. 2018;38(4): 319-331.

21. Wu YL, Herbst RS, Mann H, Rukazenkov Y, Marotti M, Tsuboi M. ADAURA: Phase III, double-blind, randomized study of osimertinib versus placebo in EGFR mutation-positive early-stage NSCLC after complete surgical resection. Clin Lung Cancer. 2018;19(4):4229-4242.

22. Jänne PA, Yang JC, Kim DW, et al. AZD9291 in EGFR inhibitor-resistant non-small-cell lung cancer. N Engl J Med. 2015;372(18):1689-1699.

23. Douillard JY, Ostoros G, Cobo M, et al. First-line gefitinib in Caucasian EGFR mutation-positive NSCLC patients: a phase-IV, open-label, single-arm study. Br J Cancer. 2014;110(1):55-62.

24. Bhatt VR, D'Souza SP, Smith LM, et al. Epidermal growth factor receptor mutational status and brain metastases in non-small-cell lung cancer. J Glob Oncol. 2017;3(3):208-217.

25. Jenkins S, Chih-Hsin Yang J, Jänne PA, et al. EGFR mutation analysis for prospective patient selection in two Phase II registration studies of osimertinib. J Thorac Oncol. 2017;12(8):1247-1256.

26. Bertranou E, Bodnar C, Dansk V. Cost-effectiveness of osimertinib in the UK for advanced EGFR-T790M non-small cell lung cancer. $J$ Med Econ. 2017;21(2):1-17.

27. Jenkins S, Yang JC, Ramalingam SS, et al. Plasma ctDNA analysis for detection of the EGFR T790M mutation in patients with advanced non-small cell lung cancer. J Thorac Oncol. 2017;12(7):1061-1070.

28. Devine A, Marignol L. Potential of amifostine for chemoradiotherapy and radiotherapy-associated toxicity reduction in advanced NSCLC: a meta-analysis. Anticancer Res. 2016;36(1):5-12.

29. Löser P, Mross B, Lapp H. Schwere Unverträglichkeitsreaktion unter Crizotinib bei ALK-positivem, metasiertem NSCLC. Pneumol. 2016;70(12):826-830.

30. Mamesaya N, Kenmotsu H, Katsumata M, Nakajima T, Endo M, Takahashi T. Osimertinib-induced interstitial lung disease after treatment with anti-PD1 antibody. Invest New Drugs. 2017;35(1):105-107.

31. Arulananda S, do H, Musafer A, Mitchell P, Dobrovic A, John T. Combination osimertinib and gefitinib in C797S and T790M EGFRmutated non-small cell lung cancer. $J$ Thorac Oncol. 2017;12(11): $1728-1732$.

32. Ricciuti B, Baglivo S, Paglialunga L, et al. Osimertinib in patients with advanced epidermal growth factor receptor T790M mutation-positive non-small cell lung cancer: rationale, evidence and place in therapy. Ther Adv Med Oncol. 2017;9(6):387-404.

33. Mccoach CE, Jimeno A. Osimertinib, a third-generation tyrosine kinase inhibitor targeting non-small cell lung cancer with EGFR T790M mutations. Drugs Today. 2016;52(10):561-568.

34. Kotake M, Murakami H, Kenmotsu H. High incidence of interstitial lung disease following practical use of osimertinib in patients who had undergone immediate prior nivolumab therapy. Ann Oncol. 2016;28(3): 669-670.

35. Chen K, Zhou F, Shen W, et al. Novel mutations on EGFR Leu792 potentially correlate to acquired resistance to osimertinib in advanced NSCLC. J Thorac Oncol. 2017;12(6):e65-e68. 
36. Chic N, Mayo-de-Las-Casas C, Reguart N. Successful treatment with gefitinib in advanced non-small cell lung cancer after acquired resistance to osimertinib. J Thorac Oncol. 2017;12(6):e78-e80.

37. Niederst MJ, Hu H, Mulvey HE, et al. The allelic context of the C797S mutation acquired upon treatment with third-generation EGFR inhibitors impacts sensitivity to subsequent treatment strategies. Clin Cancer Res. 2015;21(17):3924-3933.

38. Ercan D, Choi HG, Yun CH, et al. EGFR mutations and resistance to irreversible pyrimidine-based EGFR inhibitors. Clin Cancer Res. 2015; 21(17):3913-3923.

39. La Monica S, Cretella D, Bonelli M, et al. Trastuzumab emtansine delays and overcomes resistance to the third-generation EGFR-TKI osimertinib in NSCLC EGFR mutated cell lines. J Exp Clin Cancer Res. 2017;36(1):174-185.

40. Iams W, Chae Y. P3.02-034 Acquired resistance to osimertinib by CCDC6-RET fusion in a patient with EGFR T790M mutant metastatic lung adenocarcinoma. J Thorac Oncol. 2017;12(11):S2249-S2250.

41. Avilés-Salas A, Muñiz-Hernández S, Maldonado-Martínez HA, et al. Reproducibility of the EGFR immunohistochemistry scores for tumor samples from patients with advanced non-small cell lung cancer. Oncol Lett. 2017;13(2):912-920.
42. Shaw AT, Gandhi L, Gadgeel S, et al. Alectinib in ALK-positive, crizotinib-resistant, non-small-cell lung cancer: a single-group, multicentre, phase 2 trial. Lancet Oncol. 2016;17(2):234-242.

43. Tanimoto A, Takeuchi S, Arai S, et al. Histone deacetylase 3 inhibition overcomes $B I M$ deletion polymorphism-mediated osimertinib resistance in EGFR-mutant lung cancer. Clin Cancer Res. 2017;23(12): 3139-3149.

44. Callegari D, Ranaghan KE, Woods CJ, et al. L718Q mutant EGFR escapes covalent inhibition by stabilizing a non-reactive conformation of the lung cancer drug osimertinib. Chem Sci. 2018;9(10):2740-2749.

45. Hoyle C, Dyer M. Comments on cost-effectiveness of osimertinib for EGFR mutation-positive non-small-cell lung cancer after progression during first-line EGFR tyrosine kinase inhibitor therapy. $J$ Thorac Oncol. 2018;13(5):e83-e84.

46. Wu B, Gu X, Zhang Q. Cost-Effectiveness of osimertinib for EGFR mutation-positive non-small cell lung cancer after progression following first-line EGFR TKI therapy. J Thorac Oncol. 2018;13(2):184-193.
OncoTargets and Therapy

\section{Publish your work in this journal}

OncoTargets and Therapy is an international, peer-reviewed, open access journal focusing on the pathological basis of all cancers, potential targets for therapy and treatment protocols employed to improve the management of cancer patients. The journal also focuses on the impact of management programs and new therapeutic agents and protocols on

\section{Dovepress}

patient perspectives such as quality of life, adherence and satisfaction. The manuscript management system is completely online and includes a very quick and fair peer-review system, which is all easy to use. Visit http://www.dovepress.com/testimonials.php to read real quotes from published authors. 